\title{
Experimental analysis of eGLOSA and eGLODTA transit control strategies
}

\author{
Giulio Giorgione*, Francesco Viti, \\ Marco Rinaldi, Giorgios Laskaris \\ Faculty of Science, Technology and Communication \\ University of Luxembourg \\ Luxembourg, Luxembourg \\ giulio.giorgione.001@student.uni.lu
}

\author{
Marcin Seredynski \\ E-Bus Competence Center \\ Livange, Luxembourg
}

\begin{abstract}
Battery powered electric buses have higher energy efficiency, lower emissions and noise when compared to buses with internal combustion engines. However, due to battery charging requirements, their largescale integration into public transport operations is more complex. This study proposes a novel concept supporting said integration via new control strategies, dubbed eGLOSA and e-GLODTA. These strategies extend the existing Green Light Optimal Speed and Dwell Time Systems (GLOSA/GLODTA) to account for the specific needs of electric buses. That is, they include the goals of minimizing the energy consumption between charging stations, and maximizing available charging time. At the same time, interference with schedule requirements is minimized. The formulated heuristics are tested on a Bus Rapid Transit (BRT) corridor case study, where different scenarios-such as placement of charging stations and bus regularity-are studied to assess under which conditions each action (maintain speed, accelerate or dwell for a longer time at a stop) is beneficial. Results show that eGLOSA contributes to schedule adherence while eGLODTA allows satisfying charging time constraints.
\end{abstract}

Keywords-ITS; Electric Buses; GLOSA; GLODTA; Energy Management

\section{INTRODUCTION}

The transportation sector is one of the main contributors to climate change and air and noise pollution [1]. Such environmental impact derives from three main factors: number and length of trips, modes of transport, type of technology used. In fact, to counter the rising pollution issue of the last decades, the European Commission set a directive (2009/33/EC) aiming at a broad market introduction of environmentally friendly vehicles [2] such as electrical ones. Following the guidelines, electric cars are introduced to mitigate overall emissions. At the same time, public transport is promoted as the only space- and energy-efficient solution to serve areas with high mobility demand.

Like cars, public transport can also adopt policies to reduce its environmental impact during operations and introduce environmental friendlier vehicles. However, electric buses are still encountering operational difficulties due to their limited range and long battery charging times compared to traditional refueling process [3].

Electric buses are powered only by electricity stored in onboard batteries. Two classes of such buses differing in battery size and charging type exist. They are referred to as on-route charging and overnight charging. The buses of the first class charge medium capacity batteries in route terminals (some manufactures use additional charging at selected intermediate stops). The buses of the second class charge large battery packs (typically 200-350 kWh [4]) at night in bus depots. The way of charging determines battery size, and, operationally, bus assignment flexibility. In this article, our main focus is made on the buses pertaining to the first category.

Range improvements of electric buses can be achieved by power consumption optimization during operations, especially by means of the emerging Intelligent Transport Systems (ITS) technologies. This work focuses on two of such technologies classified as Driver Advisory Systems (DAS): Green Light Optimal Speed Advisory (GLOSA) and Green Light Optimal Dwell Time Advisory (GLODTA). GLOSA advises the driver to maintain a certain speed to pass through a green light phase without stopping [4], while GLODTA advises a prolonged dwell time in order to avoid arriving at the next signalized intersection during a red phase [5]. These strategies rely on Signal Phase and Timing (SPaT) data continuously collected from controllers placed along the route. Furthermore, real-time positions of buses in the network are accessed through Automated Vehicle Location (AVL) systems. However, the two aforementioned systems do not take into account battery charging requirements of electric buses with on route charging.

In this work, GLOSA and GLODTA are therefore extended to account for optimizing consumption and charging time at bus stops, respectively. The proposed eGLOSA instructs the driver to maintain a specific speed so that the bus traverses the next signalized intersection without stopping due to red light nor affecting signal timings, further considering the bus's own energy consumption. On the other hand, eGLODTA determines instead whether additional dwell time should be advised, considering both schedule adherence criteria and on-route battery charging needs.

The two extended DASs are therefore formulated with two main objectives: 
- Maximizing performance of on route battery charging at bus stops;

- Minimizing power consumption by increasing the probability of traversing signalized intersections without stopping at a red light.

Validation of the proposed strategies is made on a Bus Rapid Transit (BRT) line case study. Different performance measures as well as sensitivity analyses are carried out on randomized instances to assess the performances under different conditions.

The rest of the paper is structured as follows: the state of the art is introduced in Section II. Section III discusses the methodological aspects of the work, followed by the case study description in Section IV. Finally, Section V reports the results obtained and in Section VI conclusions are drawn.

\section{LITERATURE REVIEW}

\section{A. Energy Consumption in Electric Buses}

\section{1) Energy Sourcing}

Nowadays electric buses can benefit from fast charging station. They allow the bus to charge batteries connecting it to the power grid through roof-mounted fixed conductors and a pantograph mounted on the charging station. With an output DC Voltage of 500-700 $\mathrm{V}$ a charging spot can release a maximum charging power level of $300 \mathrm{~kW}$ with a frequency of $60 \pm 2$ $\mathrm{Hz} 42$ [6]. This charge a $4.8 \mathrm{kWh}$ Lithium-Ion battery with a recharge time of 6 to 10 minutes with a range of 7 to $10 \mathrm{~km}[3]$.

\section{2) Energy Consumption}

Several studies can be found related to the mechanical side or on the route management of electric powered buses, some aiming at a better understanding of the potential benefits Electric Vehicles (EV's) could bring as a cleaner propulsion system and others related instead to the integration with the current infrastructure. For example, the authors in [7] show that EVs are more efficient when driving on in-city routes than driving on freeway routes. In [8] the authors propose a theoretical equation to determine the EV performance. This equation has then been used to obtain longer running distances and good mileage with the EV. Control strategies integrated with transit system design for a mixed fleet management has further been addressed as reported in [9]. Transit system design for a mixed fleet of electric and diesel buses consists of determining the optimal composition of vehicle types, charging technologies as well as determining best locations of charging stations. Energy efficiency must be pursued not only at the electric grid level but, but also at vehicle level. That is why an approach to evaluate the energy efficiency of an electrical vehicle during a short trip has to be addressed. In [10] metrics are provided to evaluate the potential of improvement achievable via intelligent eco-driving techniques.

\section{B. ITS Based Control Strategies for Public Transport}

Traditionally the role of standalone DAS was to make traffic safer [11]. However, Connected Vehicle (CV) technology allows developing applications supporting traffic efficiency. For instance, V2I communication enables vehicles to access Signal Phase and Timing (SPaT) status from traffic signals [12] (e.g. "green signal will start in 10 seconds and will last for 30 seconds"). Access to SPaT is one of the most promising applications of V2I [13] as it enables developing cooperative variants of efficiency-oriented DAS (C-DAS) [10] and real time optimization of traffic light timing[14]. C-DAS can deliver more efficient, comfortable and automated driving [15]. Unlike in the case of signal control, which might use CV technology to collect information about the approaching vehicles, in SPaT-based C-DAS vehicles obtain signal control information in order to optimize their behavior [16]. The two SPaT-based C-DASs that are researched in literature are Green Light Optimal Speed Advisory [17]-[19] (GLOSA) and Green Light Optimal Dwell Time Advisory (GLODTA) (which applies only to PT vehicles) [5]. Both aim at mitigating stopand-go driving. GLOSA does so by providing vehicles with speed guidance, while GLODTA reaches the goal by optimizing dwell time of PT vehicles (i.e. it occasionally holds the buses longer at the near-side bus stops). Consequently, performance of traffic flow of buses is improved without the need of changing traffic signal timings (which has negative effects on general traffic). As up to $20 \%$ more fuel is used to accelerate from a full stop to a speed of 8 kilometers per hour (in case of a passenger car), there are significant benefits of moving from stop-and-go to even slow-and-go patterns.

GLOSA has been studied in several projects and field operational tests such as PREDRIVE C2X [20], DRIVEC2X [21], simTD [22], MobiTraff [5], COSMO [23], Compass4D [24]. These projects targeted Internal Combustion Engines (ICE) vehicles. GLOSA aspects for electric vehicles are studied in [25]. When it comes to bus operations, the COSMO project shows that as soon the drivers follow half of the speed recommendations time, a $20 \%$ reduction in fuel consumption is achieved [23]. Buses using exclusive lanes can obtain additional benefits from a multi-segment GLOSA variant [19]. Combination of GLOSA, GLODTA and bus priority techniques (TSP) maximizes the support that buses can receive when crossing signalized intersections. The key advantage of the speed/dwell advisory systems over TSP is they are signal nonintrusive. Hence, they do not cause externalities to the general traffic.

\section{METHODOLOGY}

\section{A. Problem Formulation}

We represent the bus infrastructure through a graph:

$$
G(E, V)
$$

where $E$ is the node set and $V$ the link set. Individual links $l_{i}$ in $E$ carry length and maximum speed information, while nodes $n_{i}$ in $V$ are subdivided into three categories: traffic light intersections, bus stops and bus terminals.

In this work, bus stops represent a key infrastructural aspect, as they interact both with the dwell time/charging dynamics (charging stations are assumed installed at bus stops) and with speed advisory dynamics (which are assumed to take 
place between the stop and the next signalized intersection). The first and the last node of the network are defined as the sole terminals. Traffic light intersections are represented as having a fixed schedule, with a-priori stages, cycle times and green/red timings.

\section{B. Assumptions}

For this study, the following assumptions are made:

- Fully dedicated public transport infrastructure (nonmixed traffic conditions);

- $\quad$ Since full charging takes approximately 6 minutes [3] it is reasonable to assume that a bus leaving the terminus will be fully charged;

- Buses are required to terminate their route with at least $10 \%$ remaining battery. This $10 \%$ is considered as a safety margin to prevent the bus to stop while still operating;

- Every calculation, such as traction force, energy and power consumption, is made considering a gross weight of $19000 \mathrm{~kg}$ for all buses [26];

- In traction force calculations, the road grade is assumed equal to zero.

- Dwell time is deterministic.

Three variables, listed in order of priority, are considered with different priority levels in our approach: battery charging, schedule adherence and energy saving. Such ranking optimizes a situation in which energy efficiency and charged batteries are needed to complete the route but, on the other hand, the adherence to the schedule is of paramount importance for the user. Three variables are considered fundamental in this study: the bus speed and acceleration $v, a$ and the travel time $t_{i}$. They form the necessary calculation core for all the decision variables. They are used as inputs for traction force, energy and power consumption and also for the energy Green Light Optimized Speed Advisory (eGLOSA) and the energy Green Light Optimal Dwell Time Advisory (eGLODTA).

\section{Energy Consumption Management}

The theoretically approached base model described in [8] has been used in the problem to find an input power function to develop the heuristic algorithms, expressed by the following formula:

$$
\mathrm{F}=\mathrm{F}_{\mathrm{a}}+\mathrm{F}_{\mathrm{r}}+\mathrm{F}_{\mathrm{k}}+\mathrm{F}_{\mathrm{i}}[\mathrm{N}]
$$

Where the running resistance $F$ is obtained as a sum of acceleration resistance $F_{a}$, rolling resistance $F_{r}$, air resistance $F_{k}$, inclination resistance $F_{i}$. The input power for an electric vehicle is function of traction force and can be obtained, once all the due coefficients and constants are known, by only observing the two variables $a$ and $v$, as described in [27]. Furthermore, to determine the energy consumption of the BEV, force and speed characteristic are evaluated through tests and then power consumption are mathematically approximated as a bilinear relation of the two as reported in [27]

$$
\mathrm{P}=\mathrm{i} \cdot \mathrm{v}+\mathrm{j} \cdot(\mathrm{F} \cdot \mathrm{v})+\mathrm{k} \cdot \mathrm{F}
$$

Once acceleration and speed are fixed, it is then possible to calculate traction force of the vehicle and the resulting power as functions of both. In order to calculate the power consumption step-by-step, speed increase has been discretized to estimate the instantaneous power consumption for all admissible $(a, v)$ configurations. The following multiobjective optimization functions (4) and (5) represent the underlying logic of the two proposed DASs:

$$
\min \mathrm{E}(\mathrm{v}(\mathrm{C}, \mathrm{g})), \mathrm{a}(\mathrm{C}, \mathrm{g}))
$$

s.t.

$$
\begin{gathered}
0 \leq \mathrm{v} \leq 14[\mathrm{~m} / \mathrm{s}] \\
0.5 \leq \mathrm{a} \leq 1.5\left[\mathrm{~m} / \mathrm{s}^{2}\right]
\end{gathered}
$$

Speed $v$ and acceleration $a$ are chosen considering the cycle and the green time of the next traffic light as described in the next section (III.D).

$$
\max E(t)
$$

with

$$
t_{d w e l l} \leq t \leq \Delta t_{i}
$$

Time $t$ is the time spent at the stop with $\Delta t_{i}=t_{i}^{a}-t_{i}^{S}$.

The eGLOSA strategy aims to minimize the energy consumption, while eGLODTA aims to maximize the energy recharge time. Moreover, both strategies account for schedule adherence, by minimizing the deviation between the actual arrival time $\left(t_{i}^{a}\right)$ and the scheduled arrival time $\left(t_{i}^{S}\right)$ of a bus at the stop. In the next subsection, the algorithms implementing said objectives are introduced.

\section{D. eGLOSA and eGLODTA control framework}

As mentioned earlier, when GLOSA is applied prior to traffic light intersections, it allows the bus to pass during the green phase advising the driver the proper speed and acceleration [28]. eGLOSA is applied in the same circumstances as GLOSA, but with an additional energy saving objective since, for electric buses, lowering overall speed and acceleration minimizes energy consumption.

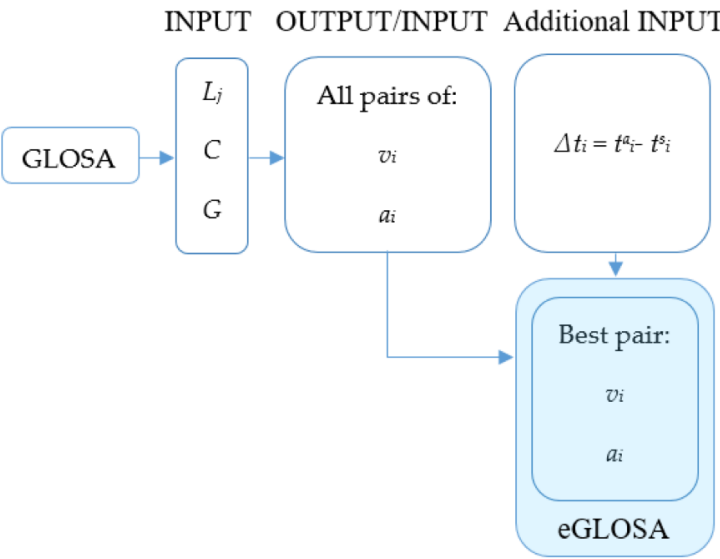

Fig. 1. eGLOSA flux diagram 
eGLOSA works as reported in Fig. 1: $L$ is the length of the link $j, C$ the cycle, $G$ the green time, $v_{i}$ the speed, $a_{i}$ the acceleration of the bus $i . \Delta t_{i}=t_{i}^{a}-t_{i}^{s}$ describes the link entering time, with $t_{i}^{a}$ the actual entering time and $t_{i}^{s}$ the scheduled entering time of bus $i$.

GLODTA is applied at a bus stop and instructs the driver to hold for a specific amount of time in order to pass through a green light phase. eGLODTA is applied in the same circumstances, including energy saving considerations. Higher acceleration upon departing from a stop leads to charge maximization: the additional dwell time can indeed be used to charge the batteries for an extra time.

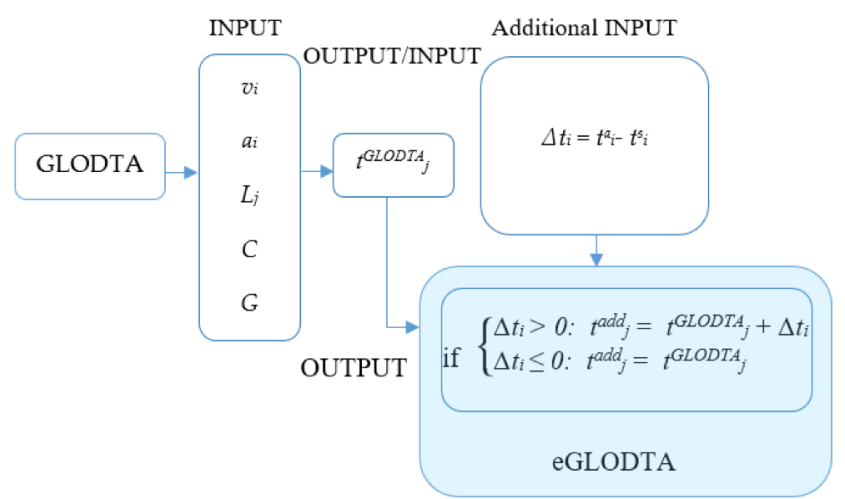

Fig. 2. eGLODTA flux diagram

eGLODTA works as reported in Fig.2: $L$ length of the link $j, C$ the cycle, $G$ the green time, $v_{i}$ the speed, $a_{i}$ the acceleration of the bus $i$. $t_{\text {GLODTA }}$ is the link $j$ travel time.

\section{CASe Study}

The case study setup begins with the generation of 100 urbanlike route instances, each with a length of 15 kilometers and with a uniform random distribution of nodes representing bus stops with fast recharging technology, bus stops without fast recharging technology and traffic lights. Secondly, a behavioral pattern is modeled to choose which DAS system has to be used for all possible combinations of network patterns, as shown in Table I.

TABLE I. Network Patterns and choices

\begin{tabular}{|c|c|}
\hline Network Patterns & Patterns' choice \\
\hline Traffic light - Bus stop & eGLOSA (from earlier calculations) \\
\hline Traffic light - Traffic light & eGLOSA \\
\hline Bus stop - Traffic light & eGLODTA \\
\hline Bus stop - Bus stop & eGLODTA (without traffic light timing) \\
\hline
\end{tabular}

The amount of randomly generated urban-like bus route instances is chosen in order to retrieve enough data and in order to make considerations on the average behavior of the controlled system (i.e. use of ITS control) and the noncontrolled one (i.e. without DAS). Generated instances are sequences of links with different lengths with a random, uniform generation of traffic lights and bus stops, always separated from the general traffic. Moreover, fast charging stations are randomly generated at stops. Cycle times, green ratios and dwell times are fixed and equal to $C_{t}=90 \mathrm{~s}, G_{r}=0.55$, $D t=25 \mathrm{~s}$ respectively. Every tested route consists of two main different scenarios composed by five sub-scenarios as shown in table II.

\begin{tabular}{|l|l|l|}
\hline \multirow{4}{*}{ Route } & \multirow{3}{*}{ Controlled } & $\Delta \mathrm{t}_{\text {init }}=-350 \mathrm{~s}$ \\
\cline { 3 - 3 } & - & $\Delta \mathrm{t}_{\text {init }}=-180 \mathrm{~s}$ \\
\cline { 3 - 3 } & Non-controlled & $\Delta \mathrm{t}_{\text {init }}=0 \mathrm{~s}$ \\
\cline { 3 - 3 } & & $\Delta \mathrm{t}_{\text {init }}=180 \mathrm{~s}$ \\
\cline { 3 - 3 } & & $\Delta \mathrm{t}_{\text {init }}=350 \mathrm{~s}$ \\
\hline
\end{tabular}

Every scenario's travel time is compared with a fixed travel time obtained with an average speed of $v_{\text {avg }}=5 \mathrm{~m} / \mathrm{s}$.

Key Performance Indicators, which are evaluated and compared among the two main scenarios, are the average difference between actual and scheduled travel time $\Delta t$ and the Battery status $E_{c}$ checked at every node.

\section{Results}

In this section eGLOSA and eGLODTA are applied to the generated routes and the results are evaluated both quantitatively and qualitatively.

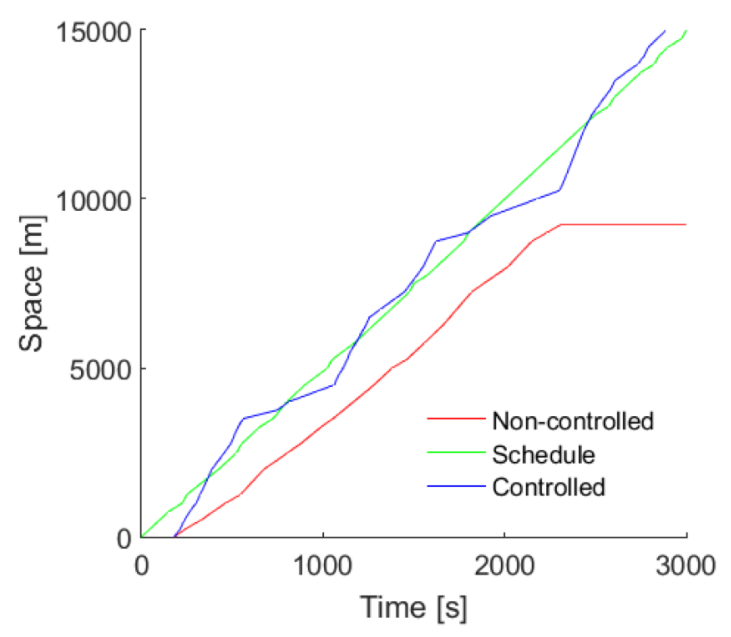

Fig. 3. Space-time profile for a single trip

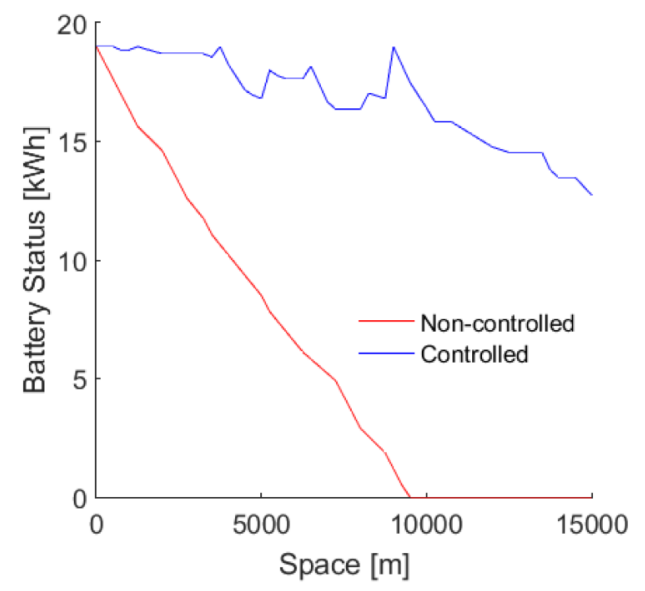

Fig. 4. Battery status profile for a DAS Controlled trip 
Fig. 3 and 4 show a space profile and a battery status profile of a trip with a bus entering late in the corridor. In Fig.3, the red line indicates buses that do not adhere to schedule. Since no recharge is possible, just before the $10^{\text {th }}$ kilometer the bus runs out of battery and remains static thereafter. On the other hand, as shown by the blue line, the bus receiving DAS advices, tends to adhere to the schedule; in Fig. 4 the battery status profile shows some increasing charge segments, in these segments the bus has been recharged at the stop following eGLODTA advices.

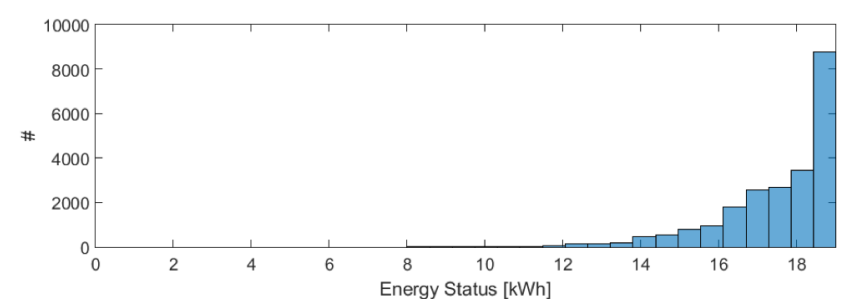

Fig. 5. Battery status checks for all the nodes of every Controlled simulation

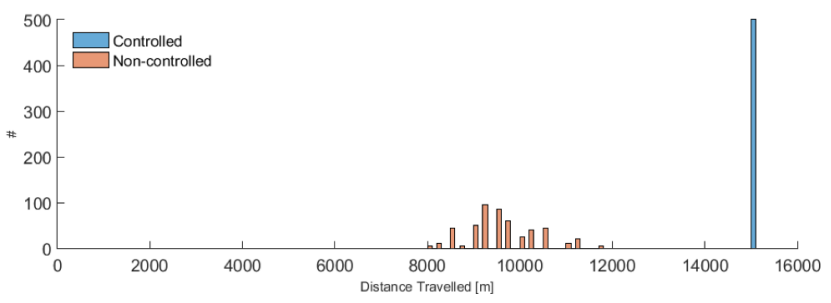

Fig. 6. Maximum distance reached by buses before running out of battery

Fig. 5 shows how a controlled trip tends to have a high battery status check through all the routes. The battery is checked at every node, as we assume lack of continuous onboard checks but, instead, discrete checkpoints performed by the roadside ITS technology.

Fig. 6 shows what is the maximum reached distance for every instance. Clearly when recharge stations are in the corridor, the bus is always able to finish to trip, on the other hand, without control the bus tends to run out of battery after 10 kilometers.

Considering schedule delay (and recovery therefrom in specific instances), Fig. 7 and Fig. 8 show how, in the controlled case, the bus tends to better adhere to the given schedule. The overall average $\Delta t$ in the Controlled case is $30.3 \mathrm{~s}$ with a standard deviation $\sigma=105.2$, meaning that the controlled systems tend to make the bus arrive slightly early, meanwhile in the Noncontrolled case the overall average $\Delta t$ is $-288.5 \mathrm{~s}$ with a standard deviation of $\sigma=288.5$, meaning that without controls the bus tends to arrive late more often than when it's controlled by the ITS.

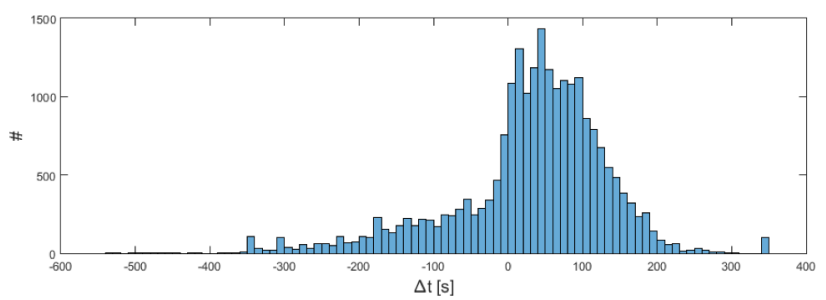

Fig. 7. $\Delta t$ distribution in the Controlled case

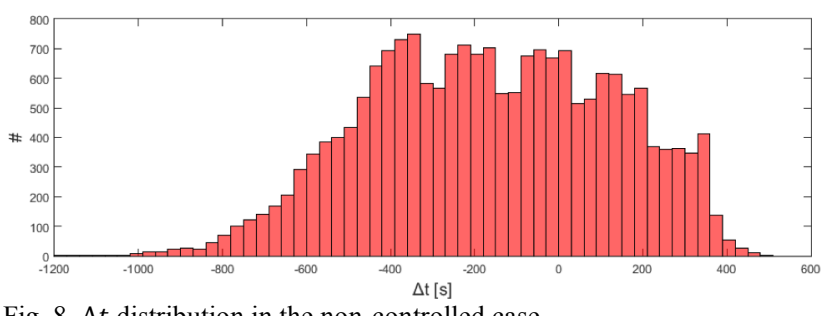

Fig. 8. $\Delta t$ distribution in the non-controlled case

\section{CONCLUSIONS}

\section{A. General Discussion}

eGLOSA and eGLODTA can clearly play an important role in achieving electric bus range extension and schedule adhering. Regarding the range extension, fast charging stations would clearly be beneficial in order to ensure route completion. Moreover, since the use of these systems also maximizes red light stop avoidance, they further contribute to range extension by lowering the energy consumption. Concerning the fast recharging station locations, a possible optimal location is given by the minimum distance travelled in a non-controlled corridor in Fig.5. Following this statistic, a fast recharging station should be located along the route before the $8^{\text {th }}$ kilometer to ensure overall trip completion.

On the schedule adherence side, the synergic use of eGLOSA and eGLODTA distinctly achieves an improvement regarding the $\Delta t_{i}$ distribution. This means that the bus tends to adhere to schedule without impacting negatively on the SPaT. Nonetheless, the standard deviation appears to be high compared to the average, which is caused by the chosen initial conditions, which are unavoidably skewing the overall distribution.

\section{B. Further Research}

Future studies will include modeling refinements, introducing for example multiple vehicle/line configurations. This might be achieved by setting different departure times at nodes differentiating upon which bus/line is currently being served; this approach would lead to develop additional constraints in order to decide which bus has to be served according to their energy state at the arrival. Such a formulation could successively be extended to an overall charging network design problem, including considerations from the perspective of the electrical grid Finally, a natural extension of this work could be testing the functionality of such control systems in shared nodes. In fact, these two DAS don't interfere with the traffic light SPaT and should therefore not cause relevant time loss on the general traffic side. 


\section{ACKNOWLEDGMENTS}

The authors would like to thank Guido Cantelmo, University of Luxembourg, for his support on the computational work on MATLAB $\AA$.

\section{REFERENCES}

[1] S. Imperatives, Report of the World Commission on Environment and Development: Our Common Future. 2016.

[2] European Parliament, "Directive 2009/33/Ec of the European Parliament and of the Council."

[3] Jean-Baptiste Gallo, Ted Bloch-Rubin, and Jasna Tomić, Peak Demand Charges and Electric Transit Buses.

[4] D. Eckhoff, B. Halmos, and R. German, "Potentials and limitations of green light optimal speed advisory systems," in Vehicular Networking Conference (VNC), 2013 IEEE, 2013, pp. 103-110.

[5] M. Seredynski and D. Khadraoui, "Complementing transit signal priority with speed and dwell time extension advisories," in Intelligent Transportation Systems (ITSC), 2014 IEEE 17th International Conference on, 2014, pp. 1009-1014.

[6] VOLVO Bus, "VOLVO Fact Sheet Opportunity Charging System." .

[7] X. Wu, D. Freese, A. Cabrera, and W. A. Kitch, "Electric vehicles' energy consumption measurement and estimation," Transp. Res. Part Transp. Environ., vol. 34, pp. 52-67, Jan. 2015.

[8] D. Tanaka, T. Ashida, and S. Minami, "An analytical method of EV velocity profile determination from the power consumption of electric vehicles," in Vehicle Power and Propulsion Conference, 2008. VPPC'08. IEEE, 2008, pp. 1-3.

[9] G. Fusco, A. Alessandrini, C. Colombaroni, and M. P. Valentini, "A Model for Transit Design with Choice of Electric Charging System," Procedia - Soc. Behav. Sci., vol. 87, pp. 234-249, Oct. 2013.

[10] W. Dib, A. Chasse, D. Di Domenico, P. Moulin, and A. Sciarretta, "Evaluation of the Energy Efficiency of a Fleet of Electric Vehicle for Eco-Driving Application,” Oil Gas Sci. Technol. - Rev. D’IFP Energ. Nouv., vol. 67, no. 4, pp. 589-599, Jul. 2012

[11] K. Bengler, K. Dietmayer, B. Farber, M. Maurer, C. Stiller, and H. Winner, "Three Decades of Driver Assistance Systems: Review and Future Perspectives,” IEEE Intell. Transp. Syst. Mag., vol. 6, no. 4, pp. 6-22, 2014

[12] M. Seredynski, G. Arnould, and D. Khadraoui, "The emerging applications of intelligent vehicular networks for traffic efficiency," 2013, pp. 101-108.

[13] California PATH Program, "Investigating the Potential Benefits of Broadcasted Signal Phase and Timing (SPaT) Data under IntelliDrive.'
[14] M. Dotoli, M. P. Fanti, and C. Meloni, "Real time optimization of traffic signal control: application to coordinated intersections," in Systems, Man and Cybernetics, 2003. IEEE International Conference on, 2003 , vol. 4, pp. 3288-3295.

[15] VDA Magazine, "Automation. from driver assistance systems to automated driving," 2015

[16] Z. Li, L. Elefteriadou, and S. Ranka, "Signal control optimization for automated vehicles at isolated signalized intersections," Transp. Res. Part C Emerg. Technol., vol. 49, pp. 1-18, Dec. 2014.

[17] Stebbins, Simon, Kim, Jiwon, Hickman, Mark, and Vu, Hai L "Characterizing Green Light Optimal Speed Advisory Trajectories for Platoon-Based Optimization," 2016, no. TRB 95th Annual Meeting Compendium of Papers.

[18] M. Seredynski, B. Dorronsoro, and D. Khadraoui, "Comparison of green light optimal speed advisory approaches," in Intelligent Transportation Systems-(ITSC), 2013 16th International IEEE Conference on, 2013, pp. 2187-2192.

[19] M. Seredynski, P. Ruiz, K. Szczypiorski, and D. Khadraoui, "Improving Bus Ride Comfort Using GLOSA-Based Dynamic Speed Optimisation," 2014, pp. 457-463.

[20] K. Katsaros, R. Kernchen, M. Dianati, D. Rieck, and C. Zinoviou, "Application of vehicular communications for improving the efficiency of traffic in urban areas: Efficiency of traffic in urban areas," Wirel. Commun. Mob. Comput., vol. 11, no. 12, pp. 1657-1667, Dec. 2011.

[21] D. Krajzewicz, L. Bieker, and J. Erdmann, "Preparing simulative evaluation of the GLOSA application," in Proceedings CD ROM 19th ITS World Congress 2012 Wien, Österreich, Paper ID: EU-00630, 2012.

[22] H. Stübing et al., "Sim TD: A Car-to-X system architecture for field operational tests [Topics in Automotive Networking]," IEEE Commun. Mag., vol. 48, no. 5, 2010.

[23] “COSMO project newsletter, number 2." 2013

[24] A. Perpey, "Compass4D D2.2 detailed technical architecture per pilot site: Bordeaux (fr)," 2014

[25] Xinkai Wu, Xiaozheng He, Guizhen Yu, A. Harmandayan, and Yunpeng Wang, "Energy-Optimal Speed Control for Electric Vehicles on Signalized Arterials," IEEE Trans. Intell. Transp. Syst., vol. 16, no. 5, pp. 2786-2796, Oct. 2015.

[26] VOLVO Bus, "VOLVO Brochure 7900E."

[27] B. Bayar, S. A. Sajadi-Alamdari, F. Viti, and H. Voos, "Impact of different spacing policies for adaptive cruise control on traffic and energy consumption of electric vehicles," in Control and Automation (MED), 2016 24th Mediterranean Conference on, 2016, pp. 1349-1354

[28] Marcin Seredynski and Djamel Khadraoui, "Combining Speed and Dwell Time Advisories for Improving Bus Ride Comfort." 